\title{
Adjusted T SPOT.TB Criteria Can Increase the Specificity of Diagnosis When Differentiating Spinal Tuberculosis From Other Spinal Infections
}

\section{Zhongjing Jiang}

Xiangya Hospital Central South University Department of Spinal Surgery

Qile Gao ( $\sim$ gaoql@csu.edu.cn )

Xiangya Hospital Central South University Department of Spinal Surgery

\section{Mingxing Tang}

Xiangya Hospital Central South University Department of Spinal Surgery

Hongqi Zhang

Xiangya Hospital Central South University Department of Spinal Surgery

Yanbing Li

Xiangya Hospital Central South University

\section{Chaofeng Guo}

Xiangya Hospital Central South University Department of Spinal Surgery

Shaohua Liu

Xiangya Hospital Central South University Department of Spinal Surgery

\section{Peng Xu}

Xiangya Hospital Central South University Department of Spinal Surgery

\section{Research Article}

Keywords: Tuberculosis, T-SPOT.TB, Spinal infection, Diagnosis

Posted Date: January 21st, 2021

DOI: https://doi.org/10.21203/rs.3.rs-142854/v1

License: (1) (1) This work is licensed under a Creative Commons Attribution 4.0 International License.

Read Full License 


\section{2 from other spinal infections}

3 Zhongjing Jiang ${ }^{1, *}$, Hongqi Zhang ${ }^{1}$, Yanbing $\mathrm{Li}^{2}$, Chaofeng Guo ${ }^{1}, \quad$ Shaohua Liu ${ }^{1}$, Peng Xu ${ }^{1}$, Mingxing Tang ${ }^{1, \#, ~ Q i l e ~ G a o ~}{ }^{1, \#}$

4 Author information

$5{ }^{1}$ Department of Spine Surgery, Xiangya Hospital, Central South University, Changsha 410008, China;

$6 \quad{ }^{2}$ Department of Clinical Laboratory, Xiangya Hospital, Central South University, Changsha, 410008, China

$7 \quad *$ First author

$8{ }^{\#}$ Correspondence to: Dr. Qile Gao and Dr. Mingxing Tang, Department of Spine Surgery, Xiangya Hospital, Central South University, No. 87, Xiangya Road,

9 Changsha 410008 , P.R. China

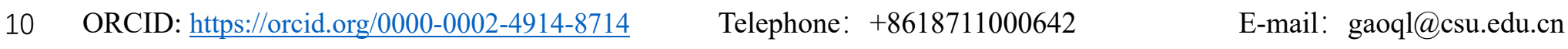

11 Keywords: Tuberculosis, T-SPOT.TB, Spinal infection, Diagnosis

12 Acknowledgment: Conflict of Interest: No benefits in any form have been or will be received from any commercial party related either directly or indirectly to the subject of

13 this manuscript. Funding: This study was supported by National Natural Science Foundation of China (No. 82072460) and Natural Science Foundation of Hunan Province,

14 China (No. 2019JJ40525, No.2019JJ40523, No.2020JJ4892). 
Background: The ability of T-SPOT.TB to differentiate Mycobacterium tuberculosis infection of the spine from other infections is little known. This study quantified the evaluated whether diagnostic performance was improved by adjusting the T-SPOT.TB assay criteria.

Methods: From January 2010 to May 2020, 147 patients with spinal infections were recruited. Peripheral blood mononuclear cells were collected, and the number of spotwere recorded. Specimen/tissue bacteriological culture was the reference standard for sensitivity and specificity. Results: There were 77 (52.4\%) participants with confirmed TB and 70 (47.6\%) with other infections. The groups were comparable in T-SPOT.TB assay results, age, sex, lesions in the segments, WBC count, CRP, procalcitonin, ESR, and TB antibodies. The sensitivity and specificity of the T-SPOT.TB assay for identifying STB was $88.3 \%$-and $40.0 \%$, respectively. On the basis of Relative operating characteristic curve (ROC) analysis and the Youden index, when we adjusted the T-SPOT.TB assay's diagnostic criteria, ESAT-6>12 or CFP-10>19, the sensitivity and specificity of the T-SPOT.TB assay for identifying STB was $83.1 \%$-and $64.3 \%$, respectively. 
Keywords: Tuberculosis, T-SPOT.TB, Spinal infection, Diagnosis

\section{INTRODUCTION}

TB cases in 2018 occurred in Southeast Asia (44\%), including China (9\%) [1]. Extrapulmonary TB accounts for 10\% of cases, of which half involve the musculoskeletal system.

The spine is the most common musculoskeletal site involved in extrapulmonary TB ( 1 to $2 \%$ of cases) [2]. Early treatment can reduce the incidence of physical disability and injury in STB, but diagnosis is extremely challenging. There are scarce bacteria in the articular effusion of lesion sites, and specimens are not easy to obtain, which reduces the positive rate of puncture fluid or joint surgical specimens [3].

The T-SPOT.TB is an assay of interferon (IFN)- $\gamma$ release from M. tuberculosis-specific effector T-cells stimulated by the Mycobacterium-specific antigens ESAT-6 (earlysecreted antigenic target 6) and CFP-10 (culture filtrate protein 10). Thus, the antigens ESAT-6 and CFP-10 have been successfully utilized to determine the presence of $M$. tuberculosis infection. The T-SPOT.TB assay is an economical, efficient, rapid, and simple laboratory technique with high sensitivity and specificity for the diagnosis of TB in patients and healthy individuals [4]. However, the effectiveness of the standard T-SPOT.TB assay for distinguishing spinal infection is unclear. 

infections from a variety of causes. A method to distinguish them is important.

In particular, data regarding the ability of the T-SPOT.TB assay to differentiate STB from other spinal infections are extremely limited; few studies have reported the application of the T-SPOT.TB assay to pinpoint the cause of spinal infection. The present study analyzed the diagnostic efficacy, sensitivity, and specificity of the T-SPOT.TB assay to distinguish STB from non-STB spinal infections. In addition, T-SPOT.TB assay diagnostic criteria were adjusted to improve its diagnostic efficacy in spinal infection.

\section{METHODS}

consent to the study.

\section{Research participants}

Patients with suspected spinal infection $(\mathrm{n}=147)$ were enrolled from January 2010 to May 2020 at Xiangya Hospital, South Centre University. Data on demographics, symptoms, and the results of each test were collected. 

months

The confirmation of STB was based on the identification of M. tuberculosis in tissues or specimens by culture or by PCR in addition to clinical, radiographic, or other supporting evidence and medical history suggestive of TB. clinical, radiographic, and other supporting tests and medical history suggested TB.

The diagnosis of other infection was made when the results of culture or PCR in tissues or specimens indicated infection other than $M$. tuberculosis, anti-TB treatment prior to surgery was ineffective, and the pulmonary and bacteriological culture of sputum was negative.

Finally, 147 patients were apportioned to three groups as follows. The confirmed STB group comprised 35 patients for whom the culture or PCR results indicated $M$. tuberculosis. The group with probable STB consisted of 42 patients in whom the pathological examination indicated TB infection. The confirmed STB and probable STB groups both received usual treatment for STB. The other infection group had culture or PCR results that suggested a cause other than $M$. tuberculosis and included 70 patients.

\section{T-SPOT.TB assay}

The T-SPOT.TB diagnosis kit was provided by Shanghai Fosun Long March Medical Science (Oxford Immunotec, United Kingdom). The T-SPOT.TB tests were 
performed in accordance with the manufacturer's instructions. Briefly, a peripheral venous blood sample was collected from each patient for the T-SPOT.TB assay to determine recorded and interpreted as recommended by the kit manufacturer, i.e., ESAT- 6 or CFP-10, with $\geq 6$ spot-forming cells regarded as T-SPOT.TB-positive. The T-SPOT.TB result was considered negative if both ESAT- 6 and CFP-10 showed $<6$ spot-forming cells. The test results were uncertain if there were $>10$ spot-forming cells in the blank control well or $<20$ spot-forming cells in the positive control well. When the results were uncertain, blood samples were retaken for another test.

\section{Statistical analysis}

The Wilcoxon signed-rank test incorporated C-reactive protein (CRP), and erythrocyte sedimentation rate (ESR) in the analysis. The statistical method used for procalcitonin (PCT), age and white blood cell (WBC) count,were independent-sample t-test. The chi-squared test was used to investigate associations between the diagnosis of spinal infection by specimen/tissue bacteriological culture or PCR results and the following: T-SPOT.TB assay result, sex, PCT, TB antibodies, and lesions in the segments.

\section{RESULTS}




\section{Patient characteristics}

Overall, 147 patients with spinal infection were registered in this study (Table 1). According to the specified classification standard, 35 (23.8\%), 42 (28.6\%), and 70 aureus; 5 Salmonella; 6 fungal; 8 bacteria other than M. tuberculosis; and 28 chronic infections, with TB eliminated (Figure 2).

\section{General indicators}

Among the diagnostic groups (confirmed STB, probable STB, and other infection), CRP and ESR were statistically significant ( $\mathrm{P}=0.03$, $\mathrm{P}=0.01$, respectively), but there were no significant differences in age, sex, lesions in the segments, WBC count, PCT (Table 2 and Table 3). Based on the chi-squared test, TB antibodies was significant differences ( $P=0.003$, Table 3), but no differences were shown in CRP, ESR, PCT, TB antibodies, WBC count, sex by logistic regression (Table 4).

\section{T-SPOT.TB assay}

Among 147 patients with valid T-SPOT.TB assay results, the sensitivity of the T-SPOT.TB assay was 88.3\% (68/77), and the specificity was 40.0\% (28/70). The 
between the results of the T-SPOT.TB assay and the diagnosis of spinal infection and in the results of the T-SPOT.TB assay among the confirmed STB, probable STB, and other infection groups $(\mathrm{P}<0.01$; Tables 3 and 4). The binary logistic regression analysis showed that for the diagnosis of spinal infection, sex, PCT, CRP, ESR, WBC count were not significant factors, but the T-SPOT.TB assay (OR 5.838, 95\% CI 1.686-20.208; P 0.005) was significant (Table 4).

\section{Adjusted T-SPOT.TB assay}

A total of 147 patients were tested using the standard T-SPOT.TB assay (positive was ESAT- 6 or CFP-10 $>6$ ). The adjusted values for the T-SPOT.TB assay were calculated by the ROC curve and Youden index, and the area under curve for ESAT-6 and CFP-10 was 0.775 and 0.785 , respectively (P<0.001), and the Youden indexes were ESAT$6=12.5$ and CFP-10=19.5. By using the adjusted-T-SPOT.TB criteria, the sensitivity of the adjusted-T-SPOT.TB assay was $83.1 \%(64 / 77)$, and the specificity was $64.3 \%(45 / 70)$. The sensitivity of the T-SPOT.TB assay to confirm STB was $91.4 \%(32 / 35)$ for the confirmed STB group and $76.2 \%$ (32/42) for the probable STB group. The false-positive rate of other infections was $35.7 \%$ (25/70). The positive predictive value (PPV) and positive likelihood ratio (PLR) were $71.9 \%$ and 2.3 , respectively (Table 5). Binary logistic regression analysis showed that the adjusted T-SPOT.TB assay was significant (OR 44.425, 95\% CI 7.828-252.123; $P<0.001)$ (Table 4 ). When we focused only on ESATsensitivity was $72.7 \%(56 / 77)$, the specificity was $84.3 \%$ (59/70), and the PPV and PLR were $83.6 \%$ and 4.6 , respectively; and when we focused only on ESAT-6>12 and CFP- 


\section{DISCUSSION}

There have been many studies of the mechanisms, diagnosis, and treatment of pulmonary TB, but until recently, extrapulmonary TB has been relatively neglected [6]. Few

Staphylococcus), and others. Bone and joint TB can cause limb deformity, limited mobility, and even paraplegia if improperly treated [7]. The T-SPOT.TB assay is a great improved by adjusting the diagnostic criteria.

In the present study, the 147 patients were apportioned to 3 groups according to the results of culture or PCR: confirmed STB, probable STB, and other infection, with 35 , 
assay was as low as $15.7 \%$ with CFP-10 $>19$, indicating that ESAT- 6 and CFP-10 should possibly be evaluated clinically to guide the differential diagnosis of spinal infections.

The adjusted T-SPOT.TB assay still had a 35.7\% false-positive rate, and the effect of ESAT-6 and CFP-10 homologs from nontuberculosis mycobacterium (NTM) on IGRAs could be a potential source of false positives; however, this has not been widely studied. ESAT-6 from Mycobacterium leprae has been shown to elicit a T-cell response from confirmed TB patients [19]. According to a study from Denmark, a higher T-SPOT positivity rate was found among individuals with NTM disease with RD1 than individuals with NTM believed to not have RD1 antigens, showing that the presence of ESAT-6 and CFP-10 in NTM affects the rates of IGRA positivity [20]. Individuals exposed to NTM may potentially have false-positive T-SPOT.TB tests if the NTM has homologous ESAT-6 or CFP-10 genes. T-SPOT.TB assays in areas with endemic NTM will likely have a lower assay specificity [16]. This conclusion is consistent with our results. Another possible reason for the false-positive rate is the effects of extraspinal tuberculosis. Prior to enrollment, medical histories were rigorously examined, and chest X-rays were compared for all participants; however, there may be patients who do not know they have TB, and some TB symptoms are not obvious and difficult to detect and diagnose.

In conclusion, the standard T-SPOT.TB assay has great sensitivity but very low specificity, and the specificity was significantly improved with almost constant sensitivity guaranteed by adjusting the diagnostic criteria of the T-SPOT assay. The Youden index calculated in our study is not necessarily the best diagnostic threshold, and a larger sample size and a closer approach to the clinical threshold are needed to use the adjusted T-SPOT.TB assay for clinical identification of spinal infections.

\section{DECLARATIONS}




\section{Ethics approval and consent to participate}

The experimental protocol was established, according to the ethical guidelines of the Helsinki Declaration and was approved by the Human Ethics Committee of Xiangya

Hospital, South Centre University and the number of 201303232. Written informed consent was obtained from individual or guardian participants. The ethics committee of

Xiangya Hospital, South Centre University approved this prospective study. All patients provided signed informed consent to the study.

\section{Consent for publication}

$150 \quad$ Not applicable

\section{Availability of data and materials}

152 All data generated or analysed during this study are included in this published article.

\section{Competing interests}

The authors declare that they have no competing interests.

\section{Funding}

This study was supported by National Natural Science Foundation of China (No. 82072460) and Natural Science Foundation of Hunan Province, China (No. 


\section{Authors' contributions}

160 Liu collected and analyzed the data. All authors reviewed the manuscript.

\section{Acknowledgements}

We appreciated Yu Li,Yijing Guo,Guanteng Yang for collecting data in the manuscript.

\section{REFERENCES}

1. World Health Organization. Global Tuberculosis Report, 2019 (WHO, 2019)

2. Dunn, R. N. , \& Ben, H. M. . (2018). Spinal tuberculosis. The Bone \& Joint Journal, 100-B(4), 425-431.

3. Zellweger, J. P. , Zellweger, A. , Ansermet, S. , Senarclens, B. D. , \& Wrighton-Smith, P. . (2005). Contact tracing using a new t-cell-based test: better correlation with tuberculosis exposure than the tuberculin skin test. Int J Tuberc Lung Dis, 9(11), 1242-1247.

4. Hadjipavlou, A. G. , Mader, J. T. , Necessary, J. T. , \& Muffoletto, A. J. . (2000). Hematogenous pyogenic spinal infections and their surgical management. Spine, 25(13), $1668-1679$.

5. Kulchavenya, E. . (2014). Extrapulmonary tuberculosis: are statistical reports accurate?. Therapeutic Advances in Infectious Disease, $2(2), 61$. 
6. Tang, Y. , Yin, L., Tang, S. , Zhang, H. , \& Lan, J. . (2017). Application of molecular, microbiological, and immunological tests for the diagnosis of bone and joint tuberculosis. Journal of Clinical Laboratory Analysis, e22260

7. Tang, X. L., Zhou, Y. X. , Wu, S. M. , Pan, Q. , Xia, B. , \& Zhang, X. L. . (2014). Cfp10 and esat6 aptamers as effective mycobacterial antigen diagnostic reagents. J Infect, 69(6), 569-580

8. Takasaki, J. , Manabe, T. , Morino, E. , Muto, Y., Hashimoto, M. , \& likura, M. , et al. (2017). Sensitivity and specificity of quantiferon-tb gold plus compared with quantiferon-tb gold in-tube and t-spot.tb on active tuberculosis in japan. Journal of infection and chemotherapy : official journal of the Japan Society of Chemotherapy, 188.

9. Theron, G. , Peter, J., Calligaro, G. , Meldau, R. , Hanrahan, C. , \& Khalfey, H., et al. (2014). Determinants of pcr performance (xpert mtb/rif), including bacterial load and inhibition, for tb diagnosis using specimens from different body compartments. entific Reports, 4.

10. Zhou, Z., Zheng, Y., \& Wang, L. (2020). A comparative study on the value of Xpert MTB/RIF and T-SPOT.TB tests in the diagnosis of bone and joint tuberculosis. Clinica chimica acta; international journal of clinical chemistry, 500, 115-119. https://doi.org/10.1016/j.cca.2019.09.026.

11. Du, F., Xie, L., Zhang, Y., Gao, F., Zhang, H., Chen, W., Sun, B., Sha, W., Fang, Y., Jia, H., Xing, A., Du, B., Zheng, L., Gao, M., \& Zhang, Z. (2018). Prospective Comparison of QFT-GIT and T-SPOT.TB Assays for Diagnosis of Active Tuberculosis. Scientific reports, 8(1), 5882. https://doi.org/10.1038/s41598-018-24285-3 
12. Wang, L., Tian, X. D., Yu, Y., \& Chen, W. (2018). Evaluation of the performance of two tuberculosis interferon gamma release assays (IGRA-ELISA and T-SPOT.TB) for diagnosing Mycobacterium tuberculosis infection. Clinica chimica acta; international journal of clinical chemistry, 479, 74-78. https://doi.org/10.1016/j.cca.2018.01.014

13. Yan, L., Xiao, H., Han, M., \& Zhang, Q. (2015). Diagnostic value of T-SPOT.TB interferon- $\gamma$ release assays for active tuberculosis. Experimental and therapeutic medicine, 10(1), 345-351. https://doi.org/10.3892/etm.2015.2463

14. Yang, Chi, Zhang, Shaojun, Yao, \& Lan, et al. (2018). Evaluation of risk factors for false-negative results with an antigen-specific peripheral blood-based quantitative t cell assay (t-spot (r).tb) in the diagnosis of active tuberculosis: a large-scale retrospective study in china. The Journal of international medical research, 46(5), $1815-1825$.

15. Han, J., Zeng, F. , \& Zhou, U. . (2016). The value of t-spot.tb in early diagnosis of tracheobronchial tuberculosis. Sarcoidosis Vasc Diffuse Lung Dis, 32(4), 336-341.

16. Agarwal, S. , Nguyen, D. T. , Lew, J. D. , Teeter, L. D. , Yamal, J. M. , \& Restrepo, B. I. , et al. (2016). Differential positive tspot assay responses to esat-6 and cfp-10 in health care workers. Tuberculosis (Edinburgh, Scotland), 101S, S83.

17. Lalvani, A., Nagvenkar, P., Udwadia, Z., Pathan, A. A., Wilkinson, K. A., Shastri, J. S., Ewer, K., Hill, A. V., Mehta, A., \& Rodrigues, C. (2001). Enumeration of T cells specific for RD1-encoded antigens suggests a high prevalence of latent Mycobacterium tuberculosis infection in healthy urban Indians. The Journal of infectious diseases, 183(3), 469-477. https://doi.org/10.1086/318081

18. Arend, S. M., Engelhard, A. C., Groot, G., de Boer, K., Andersen, P., Ottenhoff, T. H., \& van Dissel, J. T. (2001). Tuberculin skin testing compared with T-cell responses 
19. Geluk, \& A. (2002). Identification and characterization of the esat-6 homologue of mycobacterium leprae and t-cell cross-reactivity with mycobacterium tuberculosis.

PloS one, 9(4), e93986. https://doi.org/10.1371/journal.pone.0093986

Table 1. Demographic characteristics of the patients in the different categories

\begin{tabular}{lllll}
\hline & All & Confirmed STB & Probable STB & Other infection \\
\hline Subjects, $\mathrm{n}$ & $147(100 \%)$ & $35(23.8 \%)$ & $42(28.6 \%)$ & $70(47.6 \%)$ \\
Age, $\mathrm{y}$ & $50.9 \pm 15.4$ & $55.4 \pm 13.1$ & $43.6 \pm 17.9$ & $53.0 \pm 13.5$ \\
\hline Sex & & & & \\
$\quad$ Male & $82(55,8 \%)$ & $21(14.3 \%)$ & $18(12.2 \%)$ & $43(29.3 \%)$ \\
$\quad$ Female & $65(44.2 \%)$ & $14(9.5 \%)$ & $24(16.3 \%)$ & $27(18.4 \%)$ \\
\hline
\end{tabular}

Lesion location 


\begin{tabular}{lllll} 
Cervical & $2(1.4 \%)$ & 0 & 0 & $2(1.4 \%)$ \\
Cervicothoracic & $13(8.8 \%)$ & $3(2.0 \%)$ & $2(1.4 \%)$ & $8(5.4 \%)$ \\
Thoracic & $42(28.6 \%)$ & $13(8.8 \%)$ & $16(10.9 \%)$ & $13(8.8 \%)$ \\
Thoracolumbar & $7(4.8 \%)$ & $2(1.4 \%)$ & $2(1.4 \%)$ & $3(2.0 \%)$ \\
Lumbar & $70(47.6 \%)$ & $15(10.2 \%)$ & $19(12.9 \%)$ & $36(24.5 \%)$ \\
Lumbosacral & $13(8.8 \%)$ & $2(1.4 \%)$ & $3(2.0 \%)$ & $8(5.4 \%)$ \\
\hline
\end{tabular}

206

Table 2. Statistical methods and results of general indicators

\begin{tabular}{lll}
\hline & Wilcoxon signed-rank test(P-value) & Independent-sample t-test (P-value) \\
\hline CRP & 0.03 & \\
ESR & 0.01 & \\
Age & & 0.109 \\
PCT & & 0.415 \\
WBC & & 0.423 \\
\hline
\end{tabular}

208

209 Table 3. Characteristics of the STB and other infection groups, $n(\%)$ *

\begin{tabular}{llllll}
\hline & & STB $(n)$ & Other infection $(n)$ & Pearson's & $P$ \\
\hline T-SPOT.TB & Positive & $68(46.3 \%)$ & $42(28.6 \%)$ & 15.604 & $<0.001$ \\
& Negative & $9(6.1 \%)$ & $28(19.0 \%)$ & & \\
\hline
\end{tabular}




\begin{tabular}{|c|c|c|c|c|c|}
\hline \multirow[t]{2}{*}{ Sex } & Female & 38 (25.9\%) & $27(18.4 \%)$ & 1.727 & 0.189 \\
\hline & Male & $39(26.5 \%)$ & $43(29.3 \%)$ & & \\
\hline \multirow[t]{2}{*}{ PCT } & $<0.05$ & 31 (34.1\%) & $31(34.1 \%)$ & 1.922 & 0.166 \\
\hline & $>0.05$ & $10(11.0 \%)$ & $19(20.9 \%)$ & & \\
\hline \multirow[t]{2}{*}{ TB antibodies } & $\lg G(+) / \lg M(+)$ & 15 (11.1\%) & $3(2.2 \%)$ & 9.027 & 0.003 \\
\hline & $\lg G(-)$ and $\lg M(-)$ & $53(39.3 \%)$ & $64(47.4 \%)$ & & \\
\hline \multirow[t]{6}{*}{ Lesion location } & Cervical & 0 & $2(1.4 \%)$ & 9.368 & 0.095 \\
\hline & Cervicothoracic & $5(3.4 \%)$ & $8(5.4 \%)$ & & \\
\hline & Thoracic & $29(19.7 \%)$ & $13(8.8 \%)$ & & \\
\hline & Thoracolumbar & $4(2.7 \%)$ & $3(2.0 \%)$ & & \\
\hline & Lumbar & $34(23.1 \%)$ & $36(24.5 \%)$ & & \\
\hline & Lumbosacral & $5(3.4 \%)$ & $8(5.4 \%)$ & & \\
\hline
\end{tabular}

$210 *$ The STB group included both confirmed and probable STB cases.

211 Table 4. Binary logistic regression analysis of patients

\begin{tabular}{lllll}
\hline T-SPOT.TB & \multicolumn{3}{c}{ Adjusted T-SPOT.TB } \\
\cline { 2 - 3 }$(95 \% \mathrm{Cl})$ & $\mathrm{P}$ & OR $(95 \% \mathrm{Cl})$ & $\mathrm{P}$
\end{tabular}




\begin{tabular}{lllll} 
T-SPOT.TB & $5.838(1.686-20.208)$ & 0.005 & $44.425(7.828-252.123)$ & $<0.001$ \\
Sex & $0.713(0.256-1.987)$ & 0.518 & $0.674(0.196-2.312)$ & 0.530 \\
CRP & $1.003(0.986-1.019)$ & 0.762 & $1.002(0.982-1.022)$ & 0.837 \\
PCT & $1.257(0.411-3.847)$ & 0.689 & $5.675(0.937-34.354)$ & 0.059 \\
ESR & $0.999(0.980-1.019)$ & 0.943 & $1.002(0.980-1.026)$ & 0.834 \\
Antibody & $0.112(0.012-1.001)$ & 0.050 & $0.074(0.005-1.114)$ & 0.060 \\
WBC count & $1.007(0.766-1.325)$ & 0.959 & $0.887(0.636-1.237)$ & 0.479 \\
\hline
\end{tabular}

212

213

214

Table 5. Characteristics of the standard T-SPOT.TB and the adjusted-T-SPOT.TB assays

\begin{tabular}{|c|c|c|c|c|c|c|c|c|c|c|c|}
\hline & & \multicolumn{2}{|c|}{ T-SPOT.TB } & \multirow{2}{*}{\multicolumn{2}{|c|}{$\begin{array}{c}\text { Adjusted T- } \\
\text { SPOT.TB }\end{array}$}} & \multicolumn{2}{|c|}{ ESAT-6>12 Only } & \multicolumn{2}{|c|}{ CFP-10>19 $9^{\text {Only }}$} & \multirow{2}{*}{\multicolumn{2}{|c|}{$\begin{array}{c}\text { ESAT }-6>12 \text { and } \\
\text { CFP-10>19 }\end{array}$}} \\
\hline & & \multirow[b]{2}{*}{$(+)$} & \multirow[b]{2}{*}{$(-)$} & & & \multirow[b]{2}{*}{$(+)$} & \multirow[b]{2}{*}{$(-)$} & \multirow[b]{2}{*}{$(+)$} & \multirow[b]{2}{*}{$(-)$} & & \\
\hline & & & & $(+)$ & $(-)$ & & & & & $(+)$ & $(-)$ \\
\hline Spinal & $\mathrm{n}$ & 68 & 9 & 64 & 13 & 59 & 18 & 56 & 21 & 51 & 26 \\
\hline & False-negative (\%) & & 11.7 & & 16.9 & & 23.4 & & 27.3 & & 33.8 \\
\hline
\end{tabular}


Other infections

n

False-positive (\%)

42

28

60.0

25

45

22

48

35.7

31.4

11

59

62

Specificity (\%)

40.0

64.3

68.6

71.9

61.8

$\operatorname{PPV}(\%)$

1.5

2.3

72.8

2.4

15.7

11.4

T-SPOT.TB, ESAT-6>6 or CFP-10>6; Adjusted T-SPOT.TB, ESAT-6>12 or CFP-10>19; ESAT-6>9 $9^{\text {only }}$, only ESAT-6>12; CFP-10>19 only , only CFP-10>19; PPV, positive predictive value; PLR, positive likelihood ratio.

217

218 Figure 1 Flow diagram summarizing patient recruitment 


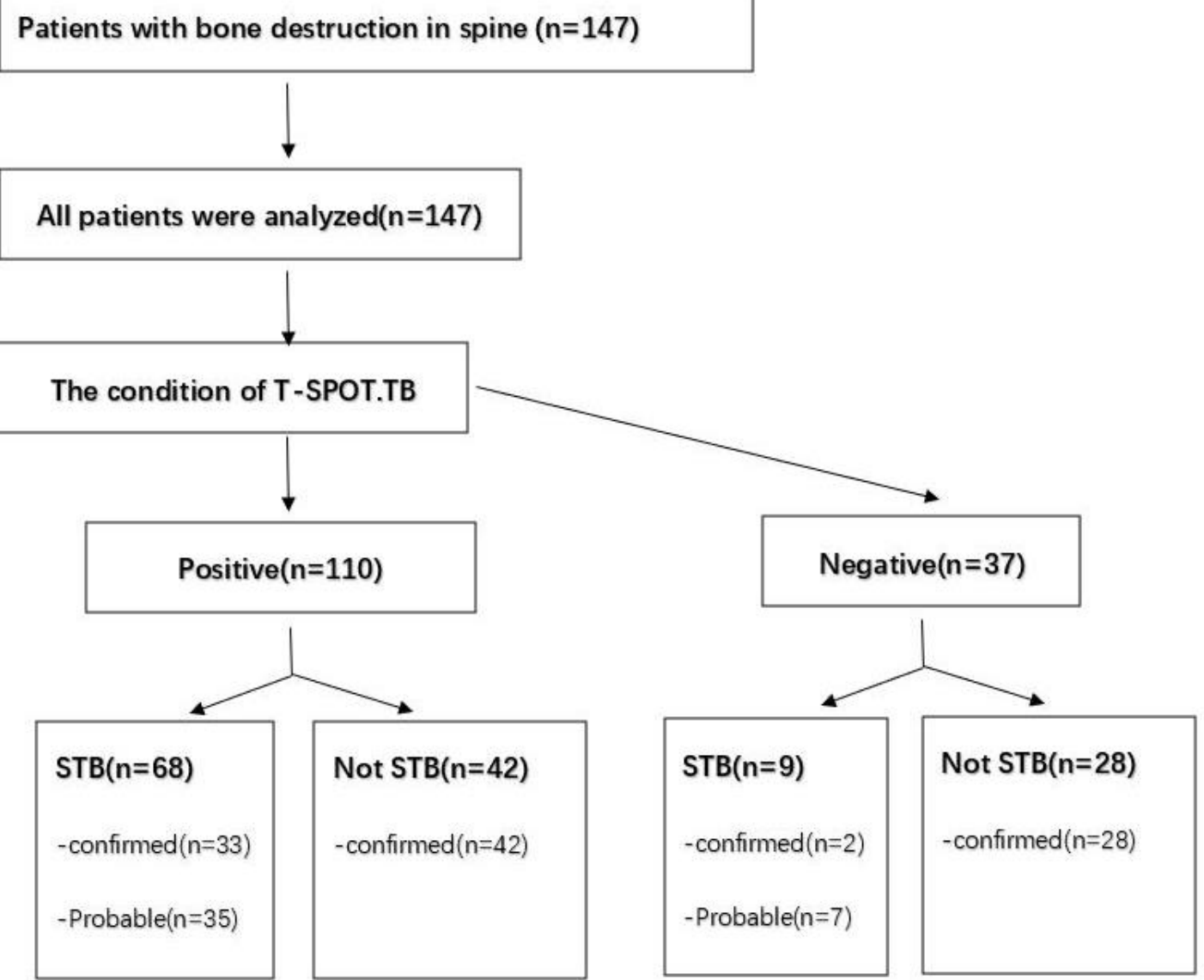



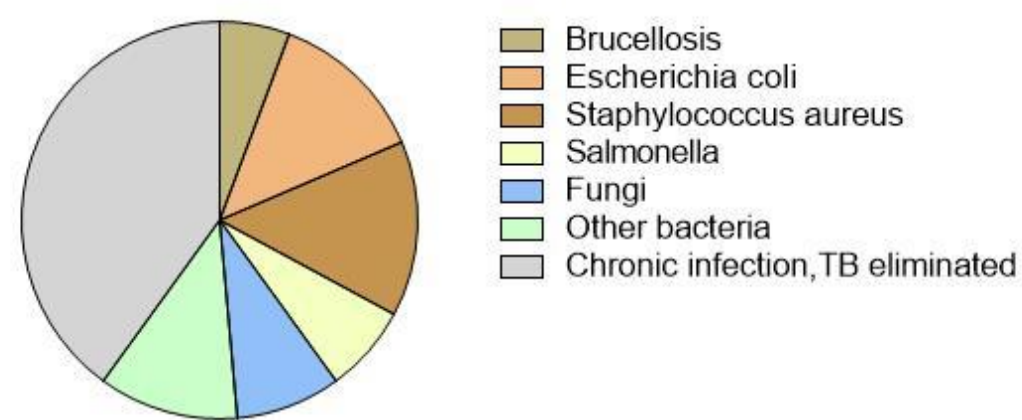

Other Infection

Figure 4 The ROC curve of ESAT- 6 and CFP-10
Figure 3 ESAT- 6 and CFP-10 spot numbers

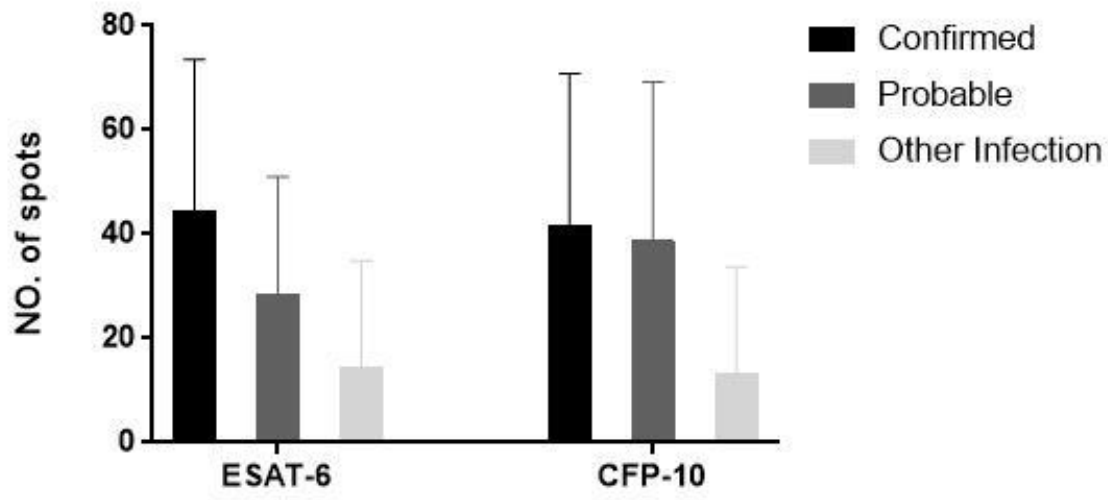




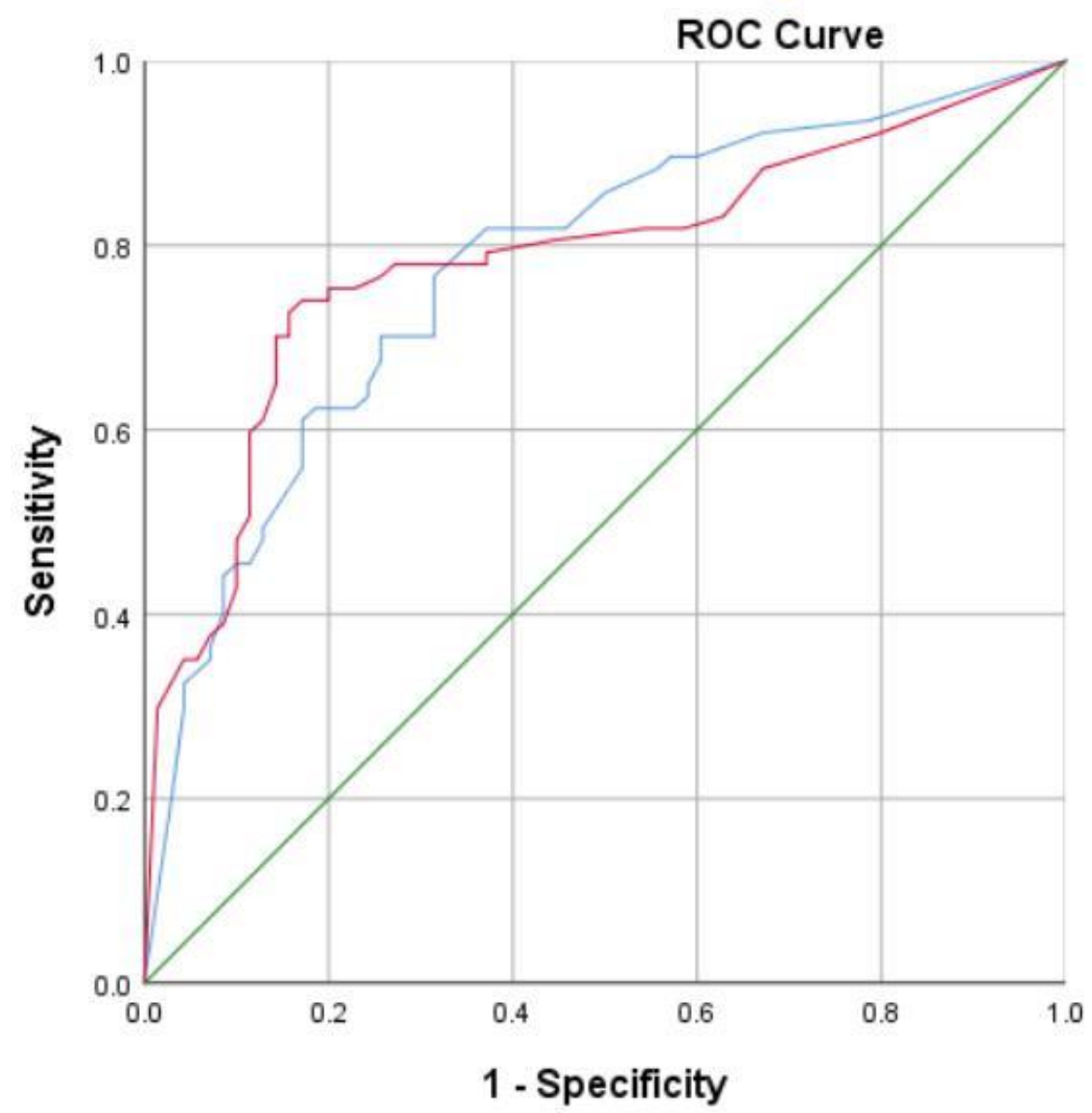

ESAT 6
- CFP-10

Baseline 
Figures

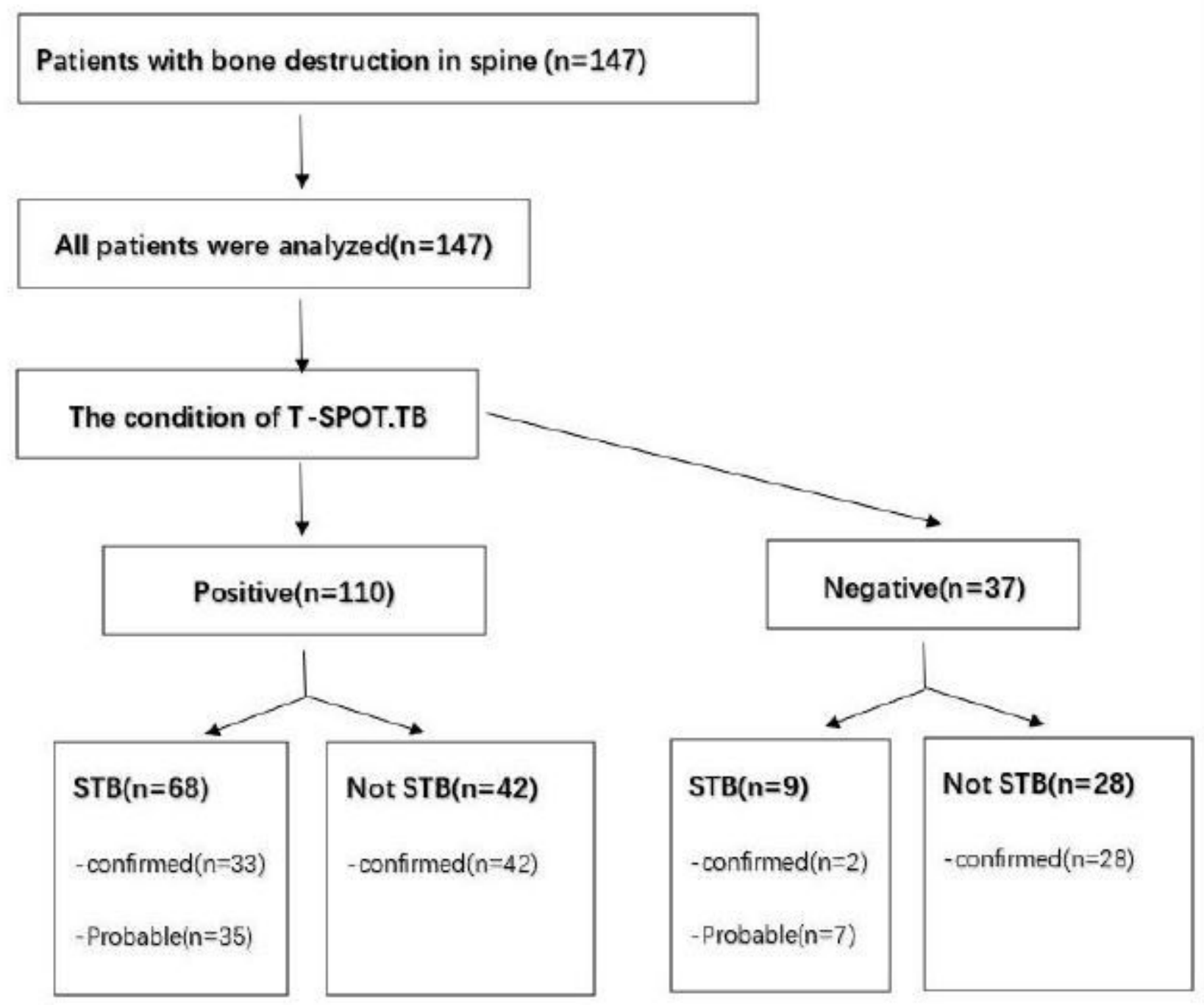

Figure 1

Flow diagram summarizi ng patient recruitment 


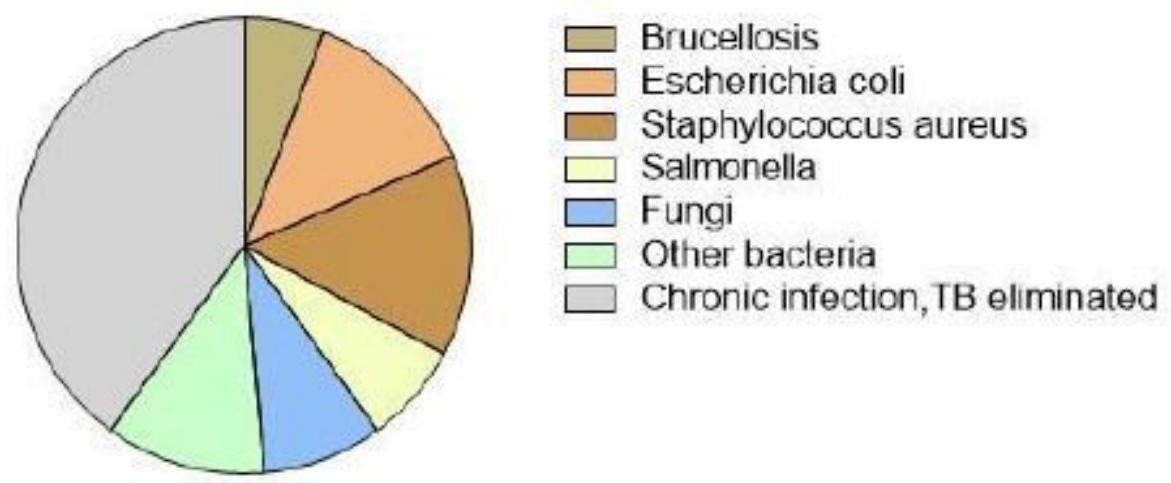

Other Infection

Figure 2

Illustration of nontuberculous spinal infections

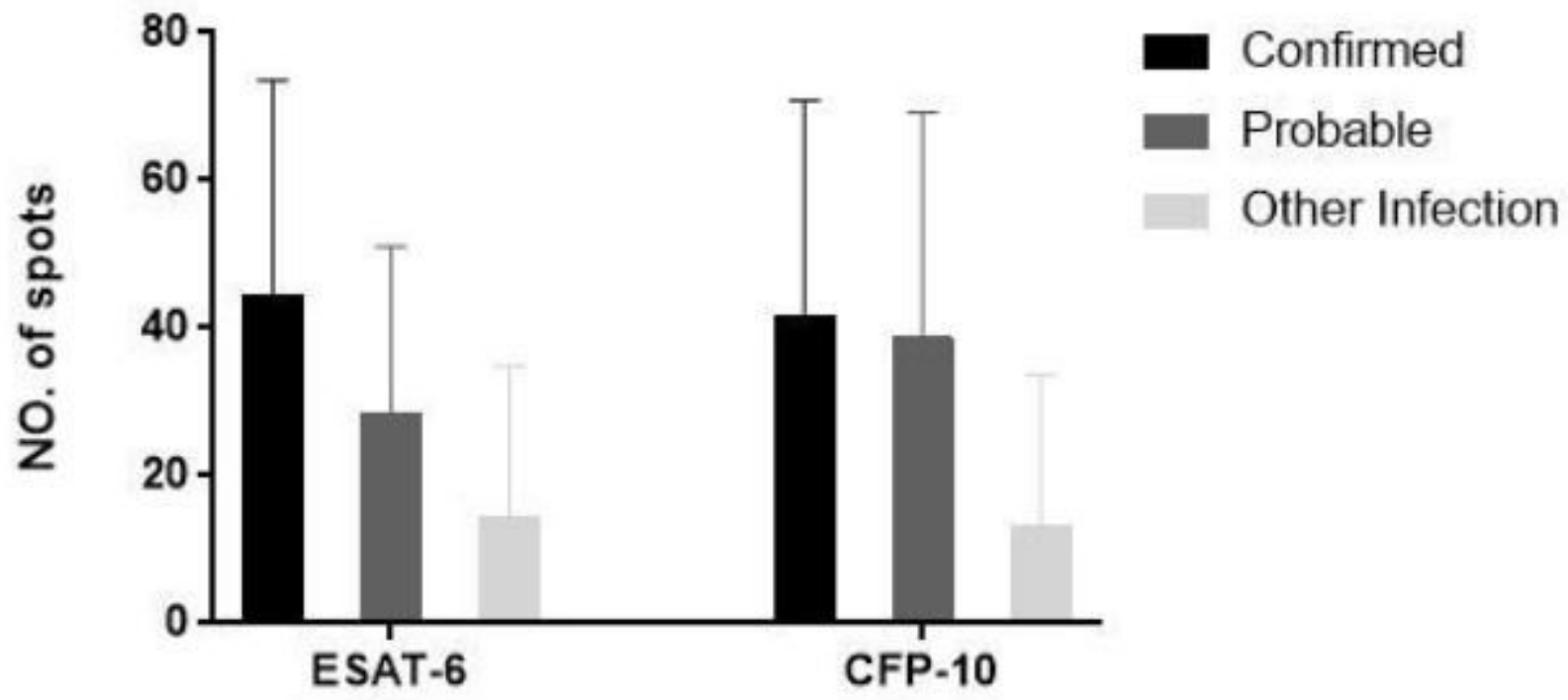

Figure 3

ESAT 6 and CFP 10 spot numbers 


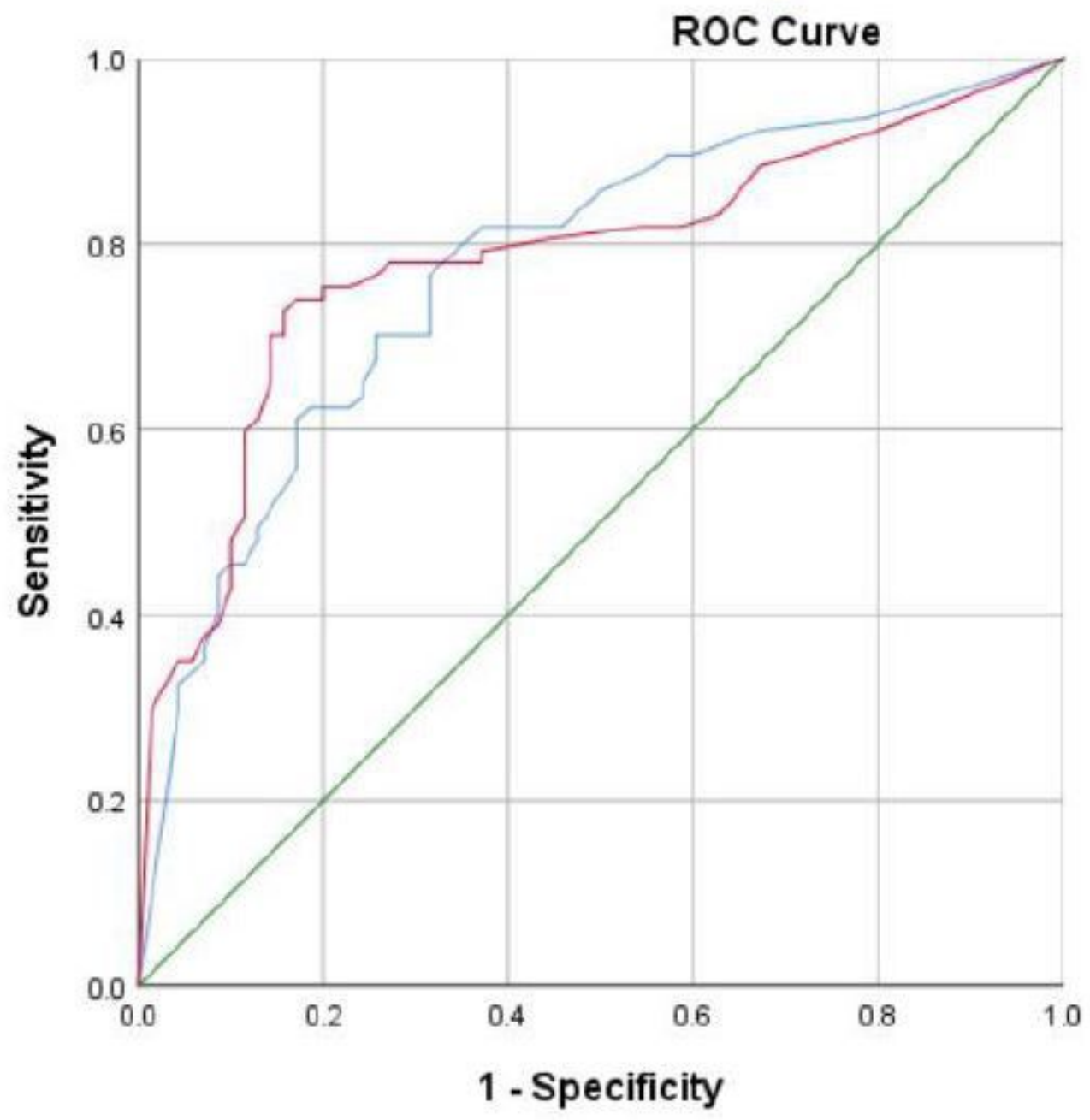

ESAT- 6

CFP.10

Baseline

Figure 4

The ROC curve of E SA T 6 and CFP 10 